\title{
Tracking optical welding through groove modes in plasmonic nano-cavities
}

\author{
J. Mertens ${ }^{1}$, A. Demetriadou ${ }^{2,3}$, R.W. Bowman ${ }^{1}$, F. Benz ${ }^{1}$, M.-E. Kleemann ${ }^{1}$, C. Tserkezis ${ }^{2}$, Y. Shi ${ }^{4}$, \\ H.Y. Yang ${ }^{4}$, O. Hess' ${ }^{3}$, J. Aizpurua', J.J. Baumberg ${ }^{*}, 1$ \\ ${ }^{1}$ NanoPhotonics Centre, Cavendish Laboratory, University of Cambridge, Cambridge, CB3 OHE, UK \\ ${ }^{2}$ Centro de Física de Materiales, Centro Mixto CSIC-UPV/EHU, and Donostia International Physics Center (DIPC), Paseo \\ Manuel Lardizabal 4, 20018 Donostia-San Sebastian, Spain \\ ${ }^{3}$ Blackett Laboratory, Department of Physics, Imperial College London, London SW7 2AZ, United Kingdom \\ ${ }^{4}$ Pillar of Engineering Product Development, Singapore University of Technology and Design, Singapore 138682, Singapore
}

We report the light-induced formation of conductive links across nanometre-wide insulating gaps. These are realised by incorporating spacers of molecules or 2D monolayers inside a gold plasmonic nanoparticle-on-mirror (NPOM) geometry. Laser irradiation of individual NPoMs controllably reshapes and tunes the plasmonic system, in some cases forming conductive bridges between particle and substrate, which shorts the nanometre-wide plasmonic gaps geometrically and electronically. Darkfield spectroscopy monitors the bridge formation in-situ, revealing strong plasmonic mode mixing dominated by clear anticrossings. Finite difference time domain simulations confirm this spectral evolution, which gives insights into the metal filament formation. A simple analytic cavity model describes the observed plasmonic mode hybridisation between tightly-confined plasmonic cavity modes and a radiative antenna mode sustained in the NPoM. Our results show how optics can reveal the properties of electrical transport across well-defined metallic nanogaps to study and develop technologies such as resistive memory devices (memristors).

Light confinement between nano-plasmonic components is now possible in nanoscale gaps and provides exquisitely-sensitive spectral information about the realm of sub-nanometre structures. The unprecedented strong field enhancement in nanoscale gaps allows access to real-time measurements of the movement of atoms and molecules. ${ }^{1-3} \mathrm{~A}$ particularly promising geometry is the nanoparticle-on-mirror (NPoM) composed of a metal nanoparticle placed above a metal mirror, which is a highly versatile system to study fundamental properties of isolated plasmonic junctions. ${ }^{2,4,5}$ Its easy and reliable fabrication allows the monitoring of nanoscale reconstructions ${ }^{1,6}$ as well as chemical reactions ${ }^{7-9}$, and the exploitation of strong plasmonic enhancements for studying surface enhanced Raman scattering (SERS) ${ }^{10-12}$ and photoluminescence $(P L)^{13,14}$. Here, we demonstrate the optically-induced formation and control of conductive bridges across gaps between the two nano-plasmonic interfaces of the NPoM geometry. Understanding and controlling the growth of such conductive connections is crucial for the development of resistive memory (or 'memristive') storage and switching devices. These devices depend on the electrochemical formation and breaking of conductive links used for ultrahigh density information storage, as well as for new generations of high-frequency tunnelling transistors. ${ }^{15,16}$ Our results show that conductive bridges forming due to the migration of metal atoms in a metallic junction can be directly observed optically, ${ }^{17}$ through the formation of several laterally-confined gap modes. By modelling these modes, a new route to the understanding and development of nanoscale devices such as memristors is provided. ${ }^{18,19}$

In nanometre-sized plasmonic cavities with gap sizes from $d=0.6 \mathrm{~nm}$ to $1.4 \mathrm{~nm}$, we show that laser irradiation can controllably change the cavity morphology at the nanoscale. We use thin spacer materials to separate Au nanoparticles (AuNPs) from a flat Au mirror surface, and form an ultra-narrow cavity. Such cavities are formed between the facets of AuNPs (Supp. Info. A), which have typical widths $w=20-30 \mathrm{~nm}$, and the underlying metal mirror. We then reshape this ultra-narrow cavity by irradiation with light, which tunes the plasmonic resonances of the system. ${ }^{20}$ The ultra-violet (UV) illumination mobilizes the outer Au atoms of the AuNP, which migrate towards the gap, thus increasing the facet size. ${ }^{7,8,25}$ While optically manipulating the cavity geometry, dark-field spectroscopy is employed to monitor the plasmonic resonances in-situ. We compare different spacer materials, of both inorganic and organic composition, and identify the formation of thin bridges within the NPoM cavity for certain spacer types. Bridge formation is observed in the spectrum

*jjb12@cam.ac.uk 
through rapid spectral shifts of the plasmonic modes, and is accompanied by strong anticrossing features in the spectral signature of the NPoM, highlighting the hierarchy of coupling mechanisms. ${ }^{22}$

In our studies, we use robust monolayers of inorganic molybdenum disulphide $\left(\mathrm{MoS}_{2}, 0.6 \mathrm{~nm}\right.$ thick) and softer

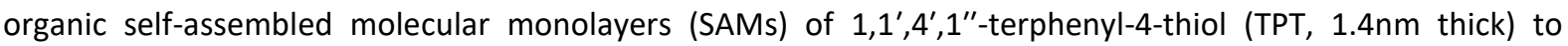
separate faceted $80 \mathrm{~nm}$ AuNPs from a flat Au surface (for fabrication details see Methods). Sparsely drop-cast AuNPs ensure that individual NPoMs can be characterised optically in a customised dark-field microscope. To activate the Au-atom migration, we irradiate individual NPoMs with a $447 \mathrm{~nm}$ continuous-wave laser of intensity $0.2 \mathrm{~mW}$, focused to a diffraction limited spot on the sample. After each irradiation step of $1 \mathrm{~s}$, the laser is turned off and a dark-field scattering spectrum of the NPoM is recorded (Fig. 1a).

The Au NPoM supports several plasmonic resonances visible in its dark-field scattering spectra when irradiating with white light at an angle of incidence of $69^{\circ}$ (see Methods for details). One resonance corresponds to a transverse ( $T$-mode) collective electron oscillation at the nanoparticle (NP), oriented parallel to the gold mirror. ${ }^{23-26}$ This mode couples only weakly to the surface modes at the underlying Au substrate. Its resonance wavelength is thus close to the resonance of an uncoupled nanoparticle at $530 \mathrm{~nm} .{ }^{27}$ In contrast, plasmon oscillations in the NP perpendicular to the surface induce image charges within the Au mirror. In this picture, the plasmonic AuNP couples to its image in the gold mirror, thus shifting the coupled resonances into the near-infra-red (NIR). Such wavelength shifts are highly sensitive to the atomistic morphology and optical properties of the gap. ${ }^{1,4,5,26,28,29}$ The alignment of the lower NP facet against the flat mirror creates an ultrathin plasmonic cavity that supports multiple 'cavity' resonances, defined by the geometry (or faceting) of the AuNP. ${ }^{1,22}$ These resonances are clearly visible in the initial scattering spectra at time $t=0$ (Fig. $1 \mathrm{~b}, \mathrm{c}$ ).

(a)

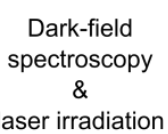

laser irradiation

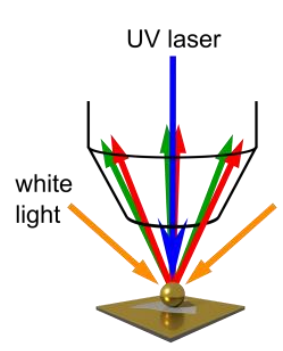

(b)
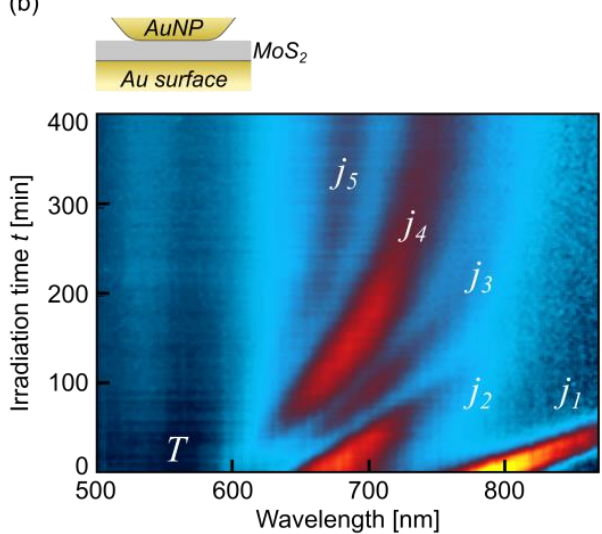

(c)

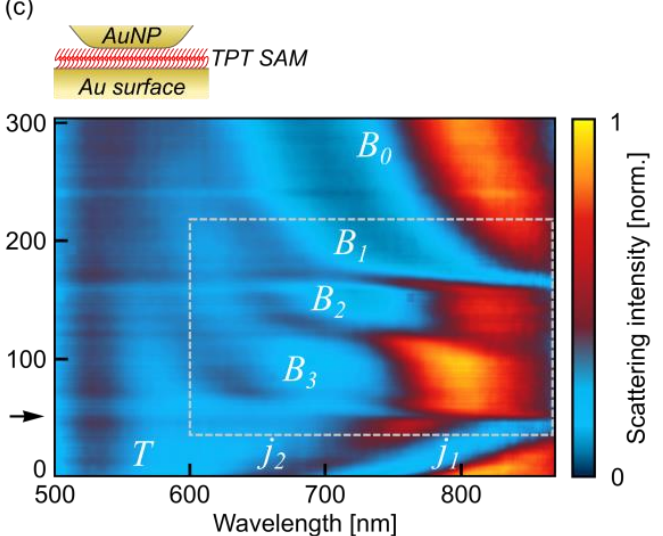

Figure 1. (a) Schematic dark-field spectroscopy of a single NPoM with laser irradiation. (b,c) Colour plot of normalised dark-field scattering spectra as a function of irradiation time for individual (b) $\mathrm{MoS}_{2} \mathrm{NPoM}_{\text {and }}$ (c) TPT NPoM. Dashed box is analysed in Figure 3; arrow highlights discontinuous transition between two regimes discussed below.

The dark-field scattering spectra of a $\mathrm{MoS}_{2}$ NPoM and a TPT NPoM are presented as a function of irradiation time (in minutes) in the colour plots of Figure $1(b, c)$. Despite the fact that the two systems are initially similar, showing two plasmonic cavity resonances $\left(j_{1}, j_{2}\right)$, they display fundamentally different behaviours after a few minutes of irradiation with the UV laser. This indicates that the Au-atom migration drastically differs between robust and soft spacers, altering in a different way the radiative properties of the cavity system. Several plasmonic resonances are visible for both systems: the transverse $T$ mode is in both cases excited but not affected by laser irradiation. In contrast the coupled resonances in the red and NIR are irreversibly shifted upon laser irradiation and fundamentally different in the two systems. Laser irradiation of $\mathrm{MoS}_{2} \mathrm{NPoMs}_{\text {(Fig. }}$ 1b) results in continuous red shifting of all surface coupled resonances $\left(j_{1}, \ldots, j_{5}\right)$, while irradiation of TPT NPoMs leads to initial red-shifts of coupled resonances $\left(j_{1}, j_{2}\right)$ followed by a sharp discontinuity (at black arrow on time axis) and subsequent blue-shifts of the plasmonic modes.

A typical $\mathrm{MoS}_{2}$ NPoM (Fig. 2a) shows two initial resonances $j_{1}$ and $j_{2}$, at $794 \mathrm{~nm}$ and $670 \mathrm{~nm}$ which both redshift linearly at rates of $1.5 \mathrm{~nm} \cdot \mathrm{min}^{-1}$ and $0.88 \mathrm{~nm} \cdot \mathrm{min}^{-1}$, respectively. Their intensity decreases after $50 \mathrm{~min}$ of UV laser irradiation and two new modes $j_{3}(650 \mathrm{~nm})$ and $j_{4}(610 \mathrm{~nm})$ emerge. Resonance $j_{3}$ disappears with further laser irradiation although mode $j_{4}$ persists until the end of the measurement $(t=400 \mathrm{~min}$ ), while after 
$100 \mathrm{~min}$ of irradiation another resonance $j_{5}$ becomes visible at $600 \mathrm{~nm}$. The initial evolution of a TPT NPoM (Fig. 1c) has a very similar signature compared to $\mathrm{MoS}_{2}$ NPoMs with two coupled resonances (at $792 \mathrm{~nm}$ and $653 \mathrm{~nm}$ ) shifting under UV irradiation at rates of $2.7 \mathrm{~nm} \cdot \mathrm{min}^{-1}$ and $2.4 \mathrm{~nm} \cdot \mathrm{min}^{-1}$. However after $50 \mathrm{~min}$ of irradiation, the sharp discontinuity changes this red-shifting behaviour to blue-shifting of multiple modes.

We thus first discuss the origin of the red-shifting behaviour, before then concentrating on the new behaviour observed with soft spacers, which will be shown to arise from conducting bridges. We find consistent behaviour for all NPoM systems with the same spacer, with minor variations in the red-shift rates and spectral positions (see below). These observations highlight the importance of the permeability of the spacer, but to account for what is going on requires electromagnetic modelling since electron microscopy of any sort damages the construct radically, changing all the spectral resonances and thus cannot be used. ${ }^{1}$

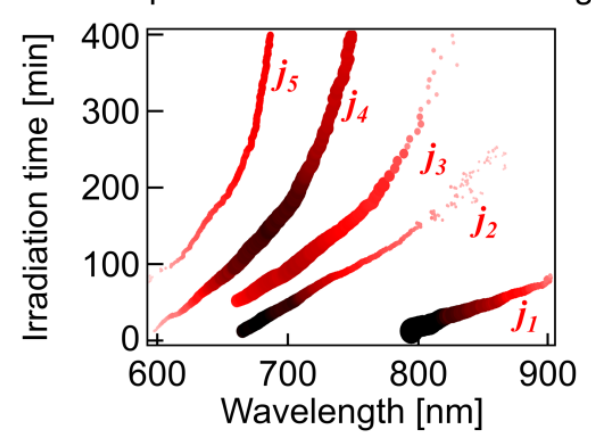

(b)

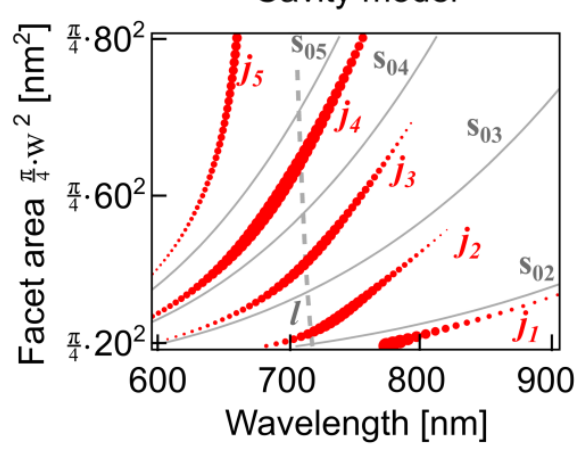

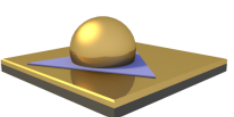

$$
\left\{\begin{array}{l}
\text { - - Antenna } \\
\text { - Cavity } \\
\text {.... Hybrid }
\end{array}\right.
$$

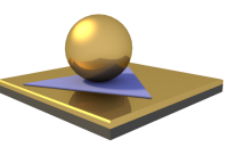

Figure 2. (a) Experimental resonant wavelengths of a $\mathrm{MoS}_{2} \mathrm{NPoM}$ vs UV irradiation time. Marker size and colour (black = high intensity, pink = low intensity) both represent the scattered mode intensity. (b) Calculated mode position for a MoS 2 NPoM as a function of facet area $\frac{\pi}{4} w^{2}$ (schematic on the right). Grey-dashed: longitudinal antenna mode $l$; grey-solid: transverse cavity modes $s_{0 i}$; red-dots: hybrid modes $j_{i}$, dot size depicts antenna-mode contribution to the hybrid mode.

\section{Inorganic 'hard' spacers}

We first consider the observed light-induced red-shifts of plasmonic resonances using a mixed cavity-antenna model. $^{1,22}$ In comparison to a single spherical scatterer, two tightly-coupled plasmonic components support both cavity modes confined to the gap between them (transverse to the axis of symmetry of the NPoM) as well as antennae modes that radiate efficiently (longitudinal to the axis of symmetry of the NPoM). These modes are mixed and radiate at specific angles depending on the exact geometry. For the NPoM system, each faceted nanoparticle couples to its image in the underlying metal mirror, forming sub-nanometre plasmonic cavities. ${ }^{1,22}$ The dispersion of cavity modes $S_{0 i}$ as a function of facet length $w$ is calculated based on the metalinsulator-metal (MIM) waveguide dispersion ${ }^{30,31}$ with Au cladding and insulator given by a monolayer of $\mathrm{MoS}_{2}$ with thickness $d=0.6 \mathrm{~nm}$ and background dielectric permittivity $\varepsilon_{d}=1.63 .{ }^{32}$ In the small gap and within a Drude approximation to describe the metal, the resonance positions $\lambda_{i}$ of the cavity modes can be analytically calculated $^{1}$ (Fig. 2 b, solid grey lines) using

$$
\lambda_{i}=\lambda_{p} \sqrt{\frac{w \varepsilon_{d}}{d \alpha_{i}}+\varepsilon_{\infty}}
$$

Here, $\lambda_{p}=137 \mathrm{~nm}$ is the plasmon wavelength of $\mathrm{Au}$ in the Drude approximation with $\varepsilon_{\infty}=10.5$ the corresponding background permittivity, and $\alpha_{i}=\alpha_{i}{ }^{\prime}-\phi$ with $\alpha_{i}^{\prime}=3.8,7.0,10.2, \ldots$ is the argument of the $i^{\text {th }}$ antinode of the cylindrical Bessel function $J_{0}$ accounting for a simplified circular facet shape, while $\phi=\pi$ is a phase-shift that accounts for the reflection of the cavity modes at the cavity edges. ${ }^{1}$ Such cavity resonances are strongly confined to the gap region but can radiate into the far-field by coupling to the main plasmonic longitudinal antenna mode $l$ (Fig. $2 b$, dashed grey line). ${ }^{1,22}$ The antenna mode is strongly radiative and originates from coupling of plasmonic charge oscillations across the AuNP with induced image charge oscillations in the Au surface (slightly blue-shifting as the NP is reshaped). New strongly-coupled hybrid modes $j_{i}$ are formed, shown as red markers in Fig. 2b. An eigenmode analysis of these hybrid modes reveals their radiative strength as a function of wavelength and cavity length, given by the antenna mode fraction of each hybrid mode. While hybrid modes close to the antenna mode radiate efficiently (larger marker size), those 
modes spectrally far from the antenna mode have a cavity-like character and radiate very weakly into the farfield.

(a)

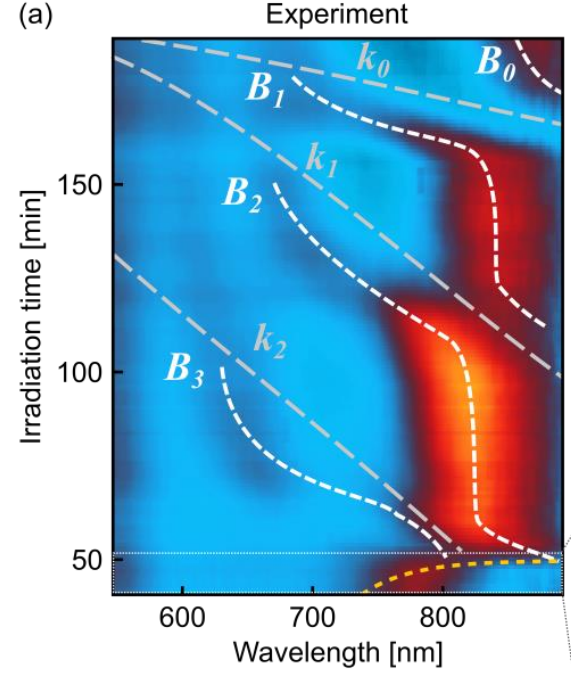

(b)

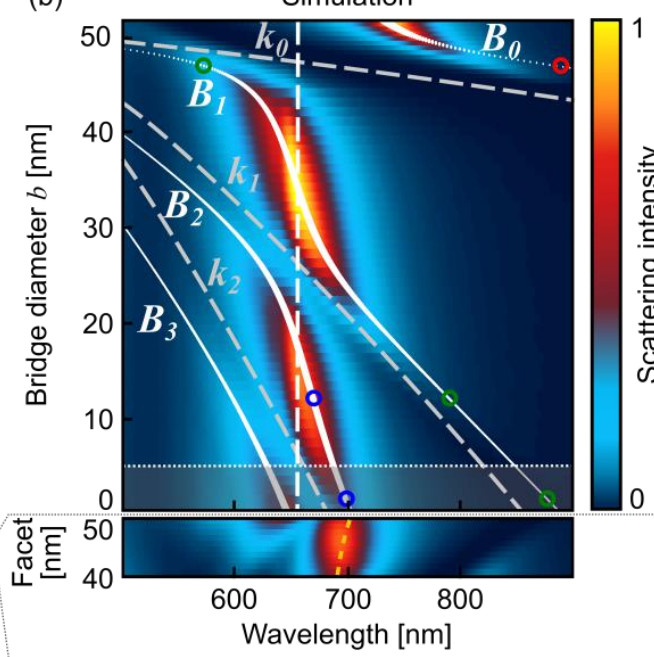

(c)

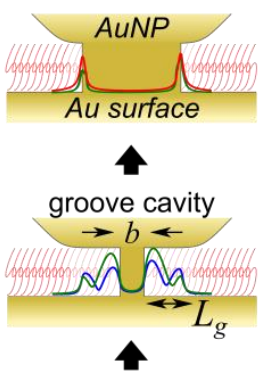

full cavity

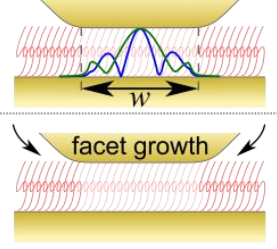

Figure 3. Irradiation of a TPT NPOM with two regimes: Bottom panel mimics the non-conductive and top panel the conductive regime. (a) Detailed experimental dark-field scattering spectra of a TPT NPoM showing tuning of coupled resonances as a function of laser irradiation. Lines are a guide to the eye, highlighting cavity modes. (b) Finite-time domain simulations (normalised colour map; yellow: high intensity, blue: low intensity) of a TPT NPoM with $w=51 \mathrm{~nm}$ facet width and central conductive bridge linking NP and surface, of diameter $b$ increased from 0 to $51 \mathrm{~nm}$ as indicated in schematic (c). Lines are analytic mode positions of cavity modes ( $k_{i}$, dashed grey), fixed antenna mode position (dashed white), and calculated hybrid mode positions (white markers, marker size gives antenna mode fraction in the hybrid modes). Grey shaded area from $b=0 \mathrm{~nm}$ to $5 \mathrm{~nm}$ (dotted white line) indicates a fast transition regime from non-conductive to conductive, not captured in the experiment dynamics. (c) Schematic of cavity model with centred cylindrical bridge. Curves show each mode intensity distribution in mid-gap for corresponding spectral positions of open circles in (b).

The observed laser-induced spectral red-shifts can then be explained by a steadily increasing size of the NP facet (Supp. Info. F). Comparison of the simple cavity model (Fig. 2b) with the experimental red-shifting results (Fig. 2a) shows excellent agreement. Particles with very small facet sizes $w<10 \mathrm{~nm}$ are not observed experimentally for $80 \mathrm{~nm}$ AuNPs (since nanoparticles are never perfectly smooth, Supp. Info. A). As previously reported, ${ }^{1}$ atoms at the AuNP surface are mobilised by laser irradiation and migrate to the gap region, thus increasing the facet size. Our results here suggest that the facet area increases linearly with time (so UV irradiation delivers a constant $\mathrm{Au}$ atom flux to the lower facet). While this may result from local heating via optical absorption, irradiating with red light where the AuNPoM has a larger absorption cross section still results in similar red-shift rates. This suggests that light can reduce barriers to surface site hopping, reduce the Au-Au bond strength, or provide optical forces (these cannot yet be distinguished). The spectral signature is thus exquisitely sensitive to minute changes of the junction morphology, which cannot yet be directly observed using electron microscopy. A deformation of particles is visible with an electron microscope only if NPoMs are irradiated for longer times at higher powers (Supp. Info. B). We emphasise that UV irradiation does not modify the $\mathrm{MoS}_{2}$ spacer layer (laser ablation only starts for $>10$ times larger power ${ }^{33}$ ). Indeed no changes in the photoluminescence and Raman scattering of $\mathrm{MoS}_{2}$ are observed before and after the laser irradiation here (Supp. Info. C).

\section{'Soft' organic molecular spacers}

Similar red-shifts from light-induced Au atom migration are first seen when UV illuminating NPoMs with softer molecular spacers, but a sudden discontinuity arises after a variable time. Two regimes can be distinguished: A non-conductive and a conductive regime. In the non-conductive regime, modes shift to the red which is explained as above by facet growth of the particle without perturbing the cavity (Fig. 3b,c lower panel). FDTD simulations using an $80 \mathrm{~nm}$ NP forming the NPoM cavity with a fixed circular facet size that increases from 
$w=40 \mathrm{~nm}$ to $w=50 \mathrm{~nm}$, provide a good understanding of spectra in the pre-conductive regime. A sharp transition in the experimental data at an irradiation time of $t=50 \mathrm{~min}$ suggests fast morphological changes while the system transitions to the conductive regime. This fast growth regime is indicated in the simulations (Fig. 3b) by a grey shaded area, in which the cavity is electronically shorted. Further simulation results (Supp.Info.N) show within this grey shaded region how Au protrusions from the NP produce fast spectral shifts leading to the groove cavity formation. The distinct threshold for the transition into the conductive regime at a facet size of $w=50 \mathrm{~nm}$ is connected to the amount of energy deposited in the cavity (Supp. Info. G).

Typically when illuminating a TPT NPoM with this power density, four distinct resonances $\left(B_{0-3}\right)$ are observed after the discontinuity (black arrow in Fig. 1c, Supp. Info. N). These resonances blue-shift and eventually merge with the spectral position of the $T$-mode at $530 \mathrm{~nm}$ for prolonged laser irradiation. Initially resonance $B_{2}$ barely shifts its spectral position under continued irradiation from $t=50 \min$ to $t=100 \mathrm{~min}$, before it then anti-crosses with $B_{1}$ which blue-shifts into the detection range from the NIR. After 160 min of laser irradiation, a similar sharp anti-crossing behaviour is again observed for mode $B_{1}$, this time interacting with the lowest order mode $B_{0}$. We now demonstrate that these spectra can be explained by metallic bridging across the insulating TPT spacers, facilitated by the absence of lateral covalent bonding in this spacer layer.

More detailed spectra of this multiple resonance shifting behaviour (Fig. 3a) show distinct blue-shifting of coupled plasmonic resonances that can be accounted for in finite different time domain (FDTD) simulations by introducing a growing Au conducting bridge between surface and nanoparticle (Fig. 3c). This conductive link has two effects: it leads to a charge transfer between nanoparticle and mirror and it strongly perturbs the cavity modes. With increasing irradiation time, it appears that such conductive bridges increase in width. As the gap capacitance increases during the initial bridge formation, the coupled modes are predicted to first red shift, as observed in experiment. Such a process resembles dc field-induced breakdown in dielectric capacitors in which fluctuations can suddenly destabilise the interface. ${ }^{34}$ To parameterise the influence of conductive bridges on the spectral far-field response of the NPoM system, FDTD simulations are performed for a TPT NPoM (see Methods for details). For these, an $80 \mathrm{~nm}$ AuNP with a fixed circular facet size of diameter $w=51 \mathrm{~nm}$ is assumed to be progressively conductively connected to the Au surface through a Au bridge in the centre of the NP facet (top panel in Fig. 3c). The diameter of the cylindrical conductive bridge is varied from $b=0 \mathrm{~nm}$ to $51 \mathrm{~nm}$, i.e. from no connection to a bridge with a size that equals the full facet (so metal fully fills the cavity formed by NP facet and Au surface, see schematics in Fig. 3c). The colour map (Fig. 3b) shows the spectral response of the connected NPoM as a function of bridge diameter $b$. With increasing bridge width, plasmonic resonances shift to the blue and mode mixing results in an anticrossing behaviour. This behaviour (Fig. 3b) matches our experimental results (Fig. 3a) very well, although the vertical irradiation timeaxis in the experiment cannot be directly linked to the diameter of the bridge in the simulations due to a nonlinear relationship between them. In addition, exact mode positions in the simulation are influenced by the size of the particle facet (Supp. Info. I). Although the facet size is kept constant in the simulations, it is possible that laser irradiation simultaneously alters both the conductive bridge size as well as the facet size. However, FDTD simulations evidence that the facet growth slows down considerably after bridges are formed (Supp. Info. K).

The anticrossing behaviour observed in the simulation and experiment can be understood by adapting the previous cavity model that assumed an unperturbed NPoM cavity (bottom schematic of Fig. 3c). We compare the simulation data with a simple analytical cavity model, assuming a perturbed cavity length $L_{g}$ that is defined by the facet diameter $w$ and the bridge diameter $b$ (centre schematic of Fig. 3c), $L_{g}=0.5(w-b)$. Resonant transverse modes of the perturbed cavity (Fig. 3c), which are referred to as groove modes ${ }^{35,36}$ in the following, are obtained for the resonance condition $L_{g}=m \cdot \lambda_{m} / 2$ and thus have a wavelength of

$$
\lambda_{m}=\frac{w-b}{m+(\varphi / 2 \pi)}
$$

where $m=0,1,2, \ldots$ is the groove cavity mode order and $\varphi$ a phase factor to account for the reflection at the particular boundary conditions of the cavity system. This phase factor is necessary for the perturbed cavity because the cavity is terminated on one end by the Au bridge whereas the other termination is set by the edge of the facet (Fig. 3c), pushing the mode towards the facet edges. The energy of these cavity modes is estimated by calculating the groove wavevector $k_{m}=2 \pi / \lambda_{m}$ and finding the corresponding energy from the metal-insulator-metal planar waveguide dispersion built of a Au-clad TPT core (Supp. Info. D). The dispersion relation of the first three cavity groove modes $k_{m}$ with fixed phase factor $\varphi=0.4 \pi$ (fitted to the simulation 
results) are plotted as a function of bridge diameter $b$ (grey dashed lines in Fig. 3b). These modes indeed shift to the blue with increasing bridge diameter because the cavity length $w-b$ reduces for larger bridge $b$.

As previously discussed, the cavity MIM modes are strongly confined to the gap region and although they do not radiate into the far-field, they mix with the longitudinal antenna mode that radiates. We fix the antenna mode at a spectral position of $680 \mathrm{~nm}$ (vertical dashed line in Fig. 3b) since this is barely perturbed by the bridging, giving spectral positions of hybrid modes calculated by solving for the eigenvalues of the coupled system (Supp. Info. D). Hybrid groove modes in Fig. 3b are white lines whose width corresponds to the strength of the antenna mode (their radiative component). This simple cavity model (white lines) fits the fullwave simulations (colour map) extremely well, thus giving a good understanding of the experimental observations as well as the simulations. Extracting the near-field distribution of resonant modes in the bridged NPoM from FDTD shows that these modes evanescently decay inside the bridge, being expelled into the grooves (Fig. 3c, Supp. Info. H). This justifies our use of a shortened cavity which arises from the perturbation in the gap induced by the bridge. It is however intriguing that the 3D problem of a bridged NPoM can be reduced to a simplified 1D cavity (Fig. 3c). Our simulations (Fig. 3b) show that first formation of a $1 \mathrm{~nm}$ diameter bridge only slightly blue-shifts the unperturbed cavity modes. To relate this to previous theoretical work, $^{37}$ we note that if such a bridge possesses bulk Au conductivity the link already has a conductance of $>100 \cdot G_{0}$. We recently showed that in this situation, the conductance is not dominating the response but the mode position is instead controlled by the kinetic inductance of the conducting wire, giving blue shifts proportional to its diameter as observed in the simulations here. ${ }^{38}$ More careful comparison of experiment with these simulations suggests that the bridge first appears with $5 \mathrm{~nm}$ diameter (horizontal dotted white line Fig. 3b) within the measurement timescale. Observing dark-field scattering spectra while optically-irradiating plasmonic constructs thus allows clear identification of the contact morphology and its dynamics, which provides crucial information to develop a theoretical understanding of what drives such nanoscale materials processes. Subsequently any desired conductive bridge width can be selected, which remains stable when the UV laser is turned off (for times in excess of many hours), and this is how dark-field spectra are recorded (see Methods Section for details).

So far in our discussion, we assumed that the bridge forms at the exact centre of a cavity. However, experimentally it is not obvious at which lateral position a bridge should form under each facet. In the specific experimental results we concentrate on above, a central location fits the simulations well. In fact FDTD simulations growing the bridge at the facet edge show a similar spectral shifting behaviour (Supp. Info. J). Again, strong mode mixing with several anticrossings of cavity and antenna modes are observed when the bridge diameter is increased. In contrast to the symmetric case with a bridge in the centre, the tuning evolution becomes distorted. Such variability in mode tuning is observed experimentally when irradiating many different NPoM with these SAM spacers (Supp. Info. L). The results of the effective central location are thus a good representation of the bridging effect in the case of Fig. 3.

There are several other parameters that influence the bridge formation, such as the exact nano-morphology and the composition of the spacer material. The robustness of the spacer material explains the differences between $\mathrm{MoS}_{2}$ and TPT spacers. While $\mathrm{MoS}_{2}$ monolayers are very robust and continuous, TPT SAMs can be affected more easily and can develop pinholes when irradiated with a laser. Only when the laser power is increased drastically, blue-shifts are found for $\mathrm{MoS}_{2}$ NPoMs similar to the ones observed for TPT NPoMs (Supp. Info. B). Furthermore, if a conductive spacer such as a SAM of biphenyl-4-4'-dithiol (BPDT) molecules is employed to realise NPoM, laser irradiation only marginally affects the coupled resonance position. No blueshifts of resonances are found and no spectral signatures that can be linked to the formation of bridges are present (Supp. Info. M). This behaviour is explained by reduced forces in the gap since the cavity between nanoparticle and Au surface is now electronically shorted by these conducting linker molecules. ${ }^{2}$

In conclusion, we present light-induced formation and real-time monitoring of nanoscale Au bridges between individual nanoparticles and a gold surface. Faceted AuNPs on top of Au mirrors form nanometre-high plasmonic cavities that can be manipulated controllably using laser irradiation. These cavities host resonances that can be effectively accounted for analytically using the MIM waveguide dispersion. Laser irradiation of individual NPoMs mobilises nanoparticle surface atoms which migrate towards the embedded spacer, thus increasing the particle facet size. Such morphological alterations tune a set of cavity resonances, which shift to the red. Pinholes induced in the spacer layer of specific materials result in the formation of thin conductive bridges that electronically and geometrically connect the AuNP with the Au substrate. The main effect of such 
bridge formation is a geometrical change in the groove cavity length. Continued laser irradiation increases the bridge width, which in turn decreases the cavity length. Resonances in the cavity are expelled from the bridge region, and shift to the blue as a function of laser irradiation and bridge width. This behaviour is observed experimentally and confirmed by FDTD simulations of the connected NPoM. A simple analytical cavity model describes well the strong coupling between longitudinal antenna mode and transverse cavity modes, and predicts plasmonic mode positions in the NPoM structure. Our results show that formation of nanometre sized wires in memristor-like geometries can be directly observed and monitored optically, and their size can be optically controlled accurately. This work allows a much better understanding of how atoms can be tracked and moved by light, opening up opportunities in sensing, quantum metrology, ultra-low power devices, and optoelectronics.

\section{Methods:}

\section{Sample fabrication:}

The Au substrates are fabricated by template stripping: a layer of $100 \mathrm{~nm} \mathrm{Au}$ is evaporated on polished silicon wafers. Small silicon substrates $\left(10 \times 10 \mathrm{~mm}^{2}\right)$ are then glued to the Au surface using epoxy glue (EPOTEK377). The samples are left on a hot-plate for $2 \mathrm{~h}$ at a temperature of $150^{\circ} \mathrm{C}$ to cure the epoxy glue. After cooling down, the top silicon substrates are gently pushed-off and Au substrates are thereby stripped off, with Au adhering to the glue and the small Si substrates. The obtained Au surface typically has a roughness of $\sim$ $0.3 \mathrm{~nm}^{39}$

Gold substrates are covered with molybdenum disulphide $\left(\mathrm{MoS}_{2}\right)$ or a self-assembled monolayer of $1,1^{\prime}, 4^{\prime}, 1^{\prime \prime}$ Terphenyl-4-thiol (TPT) molecules. Monolayers of $\mathrm{MoS}_{2}$ are grown by chemical vapour deposition (CVD) on sapphire substrates and transferred to the Au substrates employing a standard transfer method using poly(methyl methacrylate) (PMMA). ${ }^{40-43}$ Molecular layers of TPT are self-assembled on Au surfaces by immersing substrates in a $1 \mathrm{mM}$ TPT in anhydrous ethanol solution for $12 \mathrm{~h}$. The samples are then rinsed with ethanol to remove unbound excess thiols and subsequently dried with nitrogen.

Citrate stabilised, 80nm AuNPs are purchased from BBI Scientific and drop-cast for $60 \mathrm{~s}$ on the $\mathrm{MoS}_{2}$ covered substrates and for $10 \mathrm{~s}$ on the TPT coated substrates. The samples are rinsed thoroughly subsequently with deionised water to remove unbound AuNPs.

\section{Dark-field spectroscopy and laser illumination:}

Individual NPoMs are characterised optically in a customised microscope (Olympus BX51) using dark-field white-light spectroscopy in reflection geometry. Light is focused with a 100x dark-field microscope objective providing high angle illumination of up to $69^{\circ}(\mathrm{NA}=0.93)$ and collection of scattered light with a numerical aperture of $\mathrm{NA}=0.8$ (Fig. 1a). Light collection is performed in confocal geometry using a $50 \mu \mathrm{m}$ fibre as pinhole to limit the collection area on the sample ( $1 \mu \mathrm{m}$ diameter). Spectra are recorded with a cooled spectrometer (Ocean Optics QE65000) and an integration time of $1 \mathrm{~s}$.

Illumination of individual NPoMs is performed using a diode laser (Coherent cube) with $447 \mathrm{~nm}$ emission wavelength, coupled to the microscope with a single-mode fibre. Collimated laser light fills the back-focal plane of the microscope objective, thus illuminating in bright-field geometry a diffraction limited area of 360 $\mathrm{nm}$ diameter on the sample. Particles are irradiated with a power of $0.2 \mathrm{~mW}$ (power measured after the objective at the sample position), resulting in a power density of $\sim 0.8 \mathrm{~mW}_{\mu \mathrm{m}}{ }^{-2}$. Samples are placed on a motorised translation stage and each measurement step is performed as follows: first, drift is compensated by automated repositioning of the NPoM in the plane and along the focus axis. A chromatic aberration corrected dark-field spectrum is recorded (see de Nijs et al. ${ }^{32}$ for details on aberration correction) and the particle is subsequently irradiated with UV for $1 \mathrm{~s}$. After irradiation, the laser is turned-off and the process is repeated.

\section{Simulations:}

The electromagnetic response of the NPoM geometry is simulated by three dimensional Finite-Difference Time-Domain (FDTD) calculations using Lumerical FDTD Solutions v8.12.527. The AuNP is modelled as two ellipsoids merged together, resulting in an overall height of $60 \mathrm{~nm}$ and lateral diameter of $80 \mathrm{~nm}$. The height and diameter are kept constant as the morphology of the nanoparticle changes (Supp. Info. E). The optical properties of both the nanoparticle and mirror are fitted to Johnson and Christy experimental data for gold. ${ }^{44}$ The TPT-spacer is modelled with thickness of $1.1 \mathrm{~nm}$ and refractive index of $n=1.45$, while the $\mathrm{MoS}_{2}$-spacer has a thickness of $0.6 \mathrm{~nm}$ and refractive index $n=1.27$. The system is illuminated with a plane wave polarized along perpendicular to and propagating along the mirror. To ensure that fields within the nano-cavity are resolved well, extremely fine meshing parameters of $0.3 \mathrm{~nm}$ for the TPT and 0.2 for $\mathrm{MoS}_{2}$ spacer are used. 


\section{Supporting Information:}

Additional datasets of irradiated Au NPoMs as well as detailed information on the analytical model and the FDTD simulations. This material is available free of charge via the Internet at http://pubs.acs.org.

Acknowledgment: We gratefully acknowledge support from EPSRC grants EP/G060649/1, EP/L027151/1, EP/G037221/1, EPSRC NanoDTC, and ERC grant LINASS 320503. J.M. and F.B. acknowledge support from the Winton Programme of the Physics of Sustainability. J.A. acknowledge financial support from Project FIS201341184-P from MINECO and IT756-13 from the Basque Government consolidated groups.

Notes:

The authors declare no competing financial interests.

\section{References:}

(1) Sigle, D. O.; Mertens, J.; Herrmann, L. O.; Bowman, R. W.; Ithurria, S.; Dubertret, B.; Shi, Y.; Yang, H. Y.; Tserkezis, C.; Aizpurua, J.; Baumberg, J. J. ACS Nano 2015, 9 (1), 825-830.

(2) Benz, F.; Tserkezis, C.; Herrmann, L. O.; de Nijs, B.; Sanders, A.; Sigle, D. O.; Pukenas, L.; Evans, S. D.; Aizpurua, J.; Baumberg, J. J. Nano Lett. 2015, 15 (1), 669-674.

(3) Emboras, A.; Niegemann, J.; Ma, P.; Haffner, C.; Pedersen, A.; Luisier, M.; Hafner, C.; Schimmel, T.; Leuthold, J. Nano Lett. 2016, 16 (1), 709-714.

(4) Ciraci, C.; Hill, R. T.; Mock, J. J.; Urzhumov, Y.; Fernandez-Dominguez, a. I.; Maier, S. a.; Pendry, J. B.; Chilkoti, a.; Smith, D. R. Science (80-. ). 2012, 337 (6098), 1072-1074.

(5) Mock, J. J.; Hill, R. T.; Degiron, A.; Zauscher, S.; Chilkoti, A.; Smith, D. R. Nano Lett. 2008, 8 (8), 22452252.

(6) Grady, N. K.; Knight, M. W.; Bardhan, R.; Halas, N. J. Nano Lett. 2010, 10 (4), 1522-1528.

(7) Ding, T.; Mertens, J.; Sigle, D. O.; Baumberg, J. J. Adv. Mater. 2015, 27 (41), 6457-6461.

(8) Ding, T.; Sigle, D.; Zhang, L.; Mertens, J.; de Nijs, B.; Baumberg, J. ACS Nano 2015, 9 (6), 6110-6118.

(9) Lumdee, C.; Toroghi, S.; Kik, P. G. ACS Nano 2012, 6 (7), 6301-6307.

(10) Sigle, D. O.; Hugall, J. T.; Ithurria, S.; Dubertret, B.; Baumberg, J. J. Phys. Rev. Lett. 2014, 113 (8), 087402.

(11) Mubeen, S.; Zhang, S.; Kim, N.; Lee, S.; Krämer, S.; Xu, H.; Moskovits, M. Nano Lett. 2012, 12 (4), 2088 2094.

(12) Chen, S. Y.; Mock, J. J.; Hill, R. T.; Chilkoti, A.; Smith, D. R.; Lazarides, A. a. ACS Nano 2010, 4 (11), 65356546 .

(13) Lumdee, C.; Yun, B.; Kik, P. G. ACS Photonics 2014, 1 (11), 1224-1230.

(14) Kern, J.; Trügler, A.; Niehues, I.; Ewering, J.; Schmidt, R.; Schneider, R.; Najmaei, S.; George, A.; Zhang, J.; Lou, J.; Hohenester, U.; Michaelis de Vasconcellos, S.; Bratschitsch, R. ACS Photonics 2015, 2 (9), 1260-1265.

(15) Emboras, A.; Goykhman, I.; Desiatov, B.; Mazurski, N.; Stern, L.; Shappir, J.; Levy, U. Nano Lett. 2013,13 (12), 6151-6155.

(16) Hoessbacher, C.; Fedoryshyn, Y.; Emboras, A.; Melikyan, A.; Kohl, M.; Hillerkuss, D.; Hafner, C.; Leuthold, J. Optica 2014, 1 (4), 198.

(17) Marchesin, F.; Koval, P.; Barbry, M.; Aizpurua, J.; Sánchez-Portal, D. ACS Photonics 2016, 3 (2), 269277.

(18) Martino, G. Di; Tappertzhofen, S.; Hofmann, S.; Baumberg, J. Small 2016, 12 (10), 1334-1341.

(19) Celano, U.; Goux, L.; Degraeve, R.; Fantini, A.; Richard, O.; Bender, H.; Jurczak, M.; Vandervorst, W. Nano Lett. 2015, 15 (12), 7970-7975.

(20) Lassiter, J. B.; McGuire, F.; Mock, J. J.; Ciracì, C.; Hill, R. T.; Wiley, B. J.; Chilkoti, A.; Smith, D. R. Nano Lett. 2013, 13 (12), 5866-5872.

(21) Kuhlicke, A.; Schietinger, S.; Matyssek, C.; Busch, K.; Benson, O. Nano Lett. 2013, 13 (5), 2041-2046.

(22) Tserkezis, C.; Esteban, R.; Sigle, D. O.; Mertens, J.; Herrmann, L. O.; Baumberg, J. J.; Aizpurua, J. Phys. Rev. A 2015, 92 (5), 053811.

(23) Hu, M.; Ghoshal, A.; Marquez, M.; Kik, P. G. J. Phys. Chem. C 2010, 114 (16), 7509-7514.

(24) Halas, N. J.; Lal, S.; Chang, W. S.; Link, S.; Nordlander, P. Chem. Rev. 2011, 111 (6), 3913-3961.

(25) Yamamoto, N.; Ohtani, S.; Javier Garcia de Abajo, F. Nano Lett. 2011, 11 (1), 91-95.

(26) Nordlander, P.; Prodan, E. Nano Lett. 2004, 4 (11), 2209-2213.

(27) Olson, J.; Dominguez-Medina, S.; Hoggard, A.; Wang, L.-Y.; Chang, W.-S.; Link, S. Chem. Soc. Rev. 2015, 
$44(1), 40-57$.

(28) Hill, R. T.; Mock, J. J.; Urzhumov, Y.; Sebba, D. S.; Oldenburg, S. J.; Chen, S. Y.; Lazarides, A. a.; Chilkoti, A.; Smith, D. R. Nano Lett. 2010, 10 (10), 4150-4154.

(29) Esteban, R.; Aguirregabiria, G.; Borisov, A. G.; Wang, Y. M.; Nordlander, P.; Bryant, G. W.; Aizpurua, J. ACS Photonics 2015, 2 (2), 295-305.

(30) Kuttge, M.; Cai, W.; García de Abajo, F. J.; Polman, A. Phys. Rev. B 2009, 80 (3), 033409.

(31) Bozhevolnyi, S. I.; Jung, J. Opt. Express 2008, 16 (4), 2676-2684.

(32) de Nijs, B.; Bowman, R. W.; Herrmann, L. O.; Benz, F.; Barrow, S. J.; Mertens, J.; Sigle, D. O.; Chikkaraddy, R.; Eiden, A.; Ferrari, A.; Scherman, O. A.; Baumberg, J. J. Faraday Discuss. 2015, 178, 185-193.

(33) Castellanos-Gomez, a.; Barkelid, M.; Goossens, a. M.; Calado, V. E.; Van Der Zant, H. S. J.; Steele, G. a. Nano Lett. 2012, 12 (6), 3187-3192.

(34) Goldberg-Oppenheimer, P.; Steiner, U. Small 2010, 6 (11), 1248-1254.

(35) Gramotnev, D. K.; Bozhevolnyi, S. I. Nat. Photonics 2010, 4 (2), 83-91.

(36) Bermúdez-Ureña, E.; Gonzalez-Ballestero, C.; Geiselmann, M.; Marty, R.; Radko, I. P.; Holmgaard, T.; Alaverdyan, Y.; Moreno, E.; García-Vidal, F. J.; Bozhevolnyi, S. I.; Quidant, R. Nat. Commun. 2015, 6, 7883.

(37) Perez-Gonzalez, O.; Zabala, N.; Borisov, A. G.; Halas, N. J.; Nordlander, P.; Aizpurua, J. Nano Lett. 2010, 10 (8), 3090-3095.

(38) Benz, F.; de Nijs, B.; Tserkezis, C.; Chikkaraddy, R.; Sigle, D. O.; Pukenas, L.; Evans, S. D.; Aizpurua, J.; Baumberg, J. J. Opt. Express 2015, 23 (26), 33255.

(39) Benz, F.; Chikkaraddy, R.; Salmon, A.; Ohadi, H.; de Nijs, B.; Mertens, J.; Carnegie, C.; Bowman, R. W.; Baumberg, J. J. J. Phys. Chem. Lett. 2016, 7 (12), 2264-2269.

(40) Zhan, Y.; Liu, Z.; Najmaei, S.; Ajayan, P. M.; Lou, J. Small 2012, 8 (7), 966-971.

(41) Lin, Y.-C.; Zhang, W.; Huang, J.-K.; Liu, K.-K.; Lee, Y.-H.; Liang, C.-T.; Chu, C.-W.; Li, L.-J. Nanoscale 2012, $4(20), 6637-6641$.

(42) Shi, Y.; Huang, J.-K.; Jin, L.; Hsu, Y.-T.; Yu, S. F.; Li, L.-J.; Yang, H. Y. Sci. Rep. 2013, 3, 1839.

(43) Mertens, J.; Shi, Y.; Molina-Sánchez, A.; Wirtz, L.; Yang, H. Y.; Baumberg, J. J. Appl. Phys. Lett. 2014, 104 (19), 191105.

(44) Johnson, P. B.; Christy, R. W. Phys. Rev. B 1972, 1318 (1970). 
Table of contents figure:

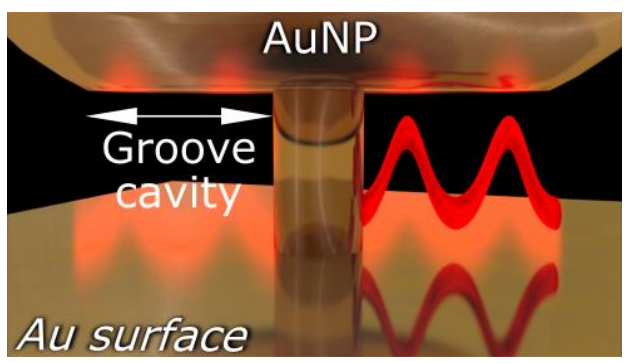

